La experiencia de ingreso a la UNLP de mayores de 25 años que no acreditan finalización del secundario

Darío Martín Estévez

Revista ES (en y sobre Educación Superior)

Vol.1, N¹-2 / Fecha de publicación: 27/12/2021

e-ISSN: $2718-6539$

https://revistas.unlp.edu.ar/ES/index

IIES - Facultad de Odontología

DOI: https://doi.org/10.24215/27186539e013

\title{
La experiencia de ingreso a la UNLP de mayores de 25 años que no acreditan finalización del secundario
}

Admission to the UNLP of people over 25-year-olds who do not prove high school completion

\section{Admissão na UNLP de pessoas maiores de 25 anos que não tenham} concluído o ensino médio

\author{
Licenciado Darío Martín Estévez \\ Secretaría de Asuntos Académicos - UNLP \\ darioestevezccu@yahoo.com.ar
}

\section{Resumen}

Nos proponemos analizar la Ordenanza 255/01 de la Universidad Nacional de La Plata, que reglamenta el Artículo 7 de la Ley $n^{\circ} 24.521$, conocida como Ley 
de Educación Superior (LES). En este artículo se estipula la posibilidad de ingreso a la Educación Superior de personas mayores de 25 años que no acrediten estudios secundarios, teniendo en cuenta para esto antecedentes y aptitudes de las personas interesadas. La Ley n² 27.204 modificó algunos aspectos sustanciales de la LES. Entre ellos, fue modificado el Artículo 7. Sin embargo, lo que respecta a la modalidad excepcional de ingreso para mayores de 25 años que no acrediten estudios secundarios, permaneció sin modificaciones.

En este artículo indagaremos sobre los supuestos teóricos en los que dicho articulado y la ordenanza que lo reglamenta se asientan; tomando como nociones centrales la inclusión educativa y valoración de esferas no formales del saber, en particular lo que mencionamos como saberes del trabajo. Entendemos estos conceptos como parte del debate sobre las políticas de acceso a la Educación Superior en la historia de nuestro país, donde el ingreso irrestricto ocupa un lugar destacado.

\begin{abstract}
We intend to analyze Ordinance 255/01 of the National University of La Plata, which regulates Article 7 of Law No. 24.521, known as the Higher Education Act (LES). This article stipulates the possibility of entry into Higher Education of persons over the age of 25 who do not accredit secondary education, taking into account the background and skills of the persons concerned. Law No. 27.204 amended some substantial aspects of the S LES. Among them, Article 7 was amended. However, as regards the exceptional mode of admission for persons over 25 years of age who do not accredit secondary studies, it remained unchanged.
\end{abstract}


In this article we will look into the theoretical assumptions in which said articulation and the ordinance regulating it are based; taking as central notions the educational inclusion and assessment of non-formal areas of knowledge, in particular what we mention as knowledge of work. We understand these concepts as part of the debate on higher education access policies in the history of our country, where unrestricted income is prominent

\section{Resumo}

Propomos analisar a Portaria 255/01 da Universidade Nacional de La Plata, que regulamenta o artigo $7^{\circ}$ da Lei $n^{\circ} 24.521$, conhecida como Lei do Ensino Superior (LES). Este artigo estipula a possibilidade de ingresso no Ensino Superior para pessoas com mais de 25 anos de idade que não tenham acreditado estudos secundários, tendo em conta esta formação e aptidões dos interessados. A Lei No. 27.204 modificou alguns aspectos substanciais do LES. Entre eles, foi modificado o artigo $7^{\circ}$, mas, quanto à forma excepcional de admissão para maiores de 25 anos que não credenciam estudos secundários, este permaneceu inalterado.

Neste artigo iremos indagar sobre os pressupostos teóricos em que se baseiam os referidos artigos e a portaria que os regulamenta; tomando como noções centrais a inclusão educacional e a valorização das esferas não formais do conhecimento, em particular o que chamamos de saber do trabalho. Entendemos esses conceitos como parte do debate sobre as políticas de acesso ao Ensino Superior na história de nosso país, onde o acesso irrestrito ocupa lugar de destaque.

\section{Palabras clave}


Ingreso, Acreditación, Educación Secundaria, Finalización.

\section{Keywords}

Income, Accreditation, Secondary Education, Completion

\section{Palavras chave}

Admissão, Credenciamento, Ensino médio, Conclusão.

\section{Introducción}

En el presente artículo analizaremos la aplicación de la Ordenanza 255/01 de la Universidad Nacional de La Plata, que reglamenta el Artículo 7 de la Ley $n^{\circ}$ 24.521, conocida como Ley de Educación Superior (LES). Allí se estipula una modalidad de ingreso específica para aspirantes que tengan más de 25 años de edad y no acrediten estudios secundarios finalizados.

El área encargada de la aplicación de esta Ordenanza es la Secretaría de Asuntos Académicos de la UNLP, ubicada dentro de la órbita de la Vicepresidencia Académica.

Dicha Secretaría tiene como funciones primarias asistir a la Presidencia en todo lo relativo a la actividad académica de la Universidad, promover la construcción de políticas académicas transversales en el ámbito de la UNLP a través del intercambio de criterios y experiencias entre las distintas dependencias y Facultades, impulsar el desarrollo de políticas académicas que promuevan condiciones de igualdad de derechos y posibilidades para el acceso, permanencia y egreso en los distintos niveles de formación de la UNLP, contribuir al diseño e implementación de nuevas carreras y opciones de formación en el ámbito de la UNLP, atendiendo a las necesidades sociales, 
contribuir al desarrollo de la capacitación y formación de posgrado de les docentes de la UNLP, consolidar y ampliar los vínculos con otras instituciones, jurisdicciones y niveles del sistema educativo y favorecer la inserción de la UNLP en redes nacionales e internacionales de formación académica.

En este sentido, dentro de esta estrategia general, nos interesar destacar lo referido a la promoción de un ingreso masivo, ya que se busca "posicionar a la UNLP como un destino posible en el imaginario de todos los sectores de la región" (Fernando Tauber, 2016, p.16), sobre todo les mayores de 25 años sin título secundario, que pueden ingresar a las Universidades Nacionales siempre que demuestren "que tienen preparación o experiencia laboral acorde con los estudios que se proponen iniciar, así como aptitudes y conocimientos suficientes para cursarlos satisfactoriamente" (Ley de Educación Superior, artículo 7).

La Ordenanza que establece los mecanismos para garantizar la evaluación de aptitudes de dichos aspirantes fue aprobada por la UNLP en el año 2001. Sin embargo, en dieciséis años de aplicación de esta Ordenanza, muy pocos aspirantes mayores de 25 años lograron ingresar a la UNLP a través de los mecanismos que ella estipula: la enorme mayoría de les aspirantes no lograron aprobar las evaluaciones requeridas para ingresar.

\section{Ingreso a las Universidades Nacionales en la historia argentina}

En Argentina el acceso a la educación superior es abierto e irrestricto por Ley desde 2015, y por decreto desde 1949. Este es uno de los elementos que permite explicar la masividad de nuestro sistema universitario. Sin embargo, este elemento que aparece como dado en nuestra actualidad no es una constante en nuestra historia ni mucho menos un factum en todo el mundo. 
A nivel mundial, el proceso de masificación de una institución tradicionalmente aristocrática como la Universidad es muy reciente: lo podemos rastrear sobre todo a partir de 1945, hace apenas 70 años. En el marco de los cambios en el capitalismo de la segunda posguerra, la Universidad atravesó un enorme e inédito proceso de crecimiento cuantitativo en todo el mundo. Asistimos por primera vez en la historia a la existencia de una explosión de la matrícula universitaria y una fuerte masificación de la educación superior (Buchbinder, 2005).

En nuestro país la Universidad había sido refundada con la Reforma Universitaria de 1918 como una institución laica, autónoma y cogobernada; pero no necesariamente de acceso irrestricto. Este proceso que puso todo en discusión, tuvo como eje central la necesidad de democratización de la Universidad. Como dice Chiroleu, la lucha de la juventud de la Reforma "apunta especialmente a expandir el ingreso de las clases medias, las cuales dificultosamente se estaban abriendo paso en la institución." (Chiroleu, 2009, p. 56). En ese momento existió el debate sobre la posibilidad de avanzar en la gratuidad universitaria, promovido por los reformistas Dante Ardigó y Gabriel del Mazo. Sin embargo, en los postulados aprobados por el Congreso de la Federación Universitaria Argentina en julio de 1918, este tema no fue incluido (Buchbinder, 2005).

Hubo que esperar al peronismo para que la Universidad se convirtiese finalmente en gratuita y de acceso irrestricto. El Plan Quinquenal 1947-1951 planteaba la gratuidad universitaria, aunque no su acceso irrestricto, pero ambas posibilidades quedaron afuera de la Ley Universitaria de 1947, que habilitaba la posibilidad del arancelamiento. Es en 1949 cuando Juan Domingo Perón firma el decreto que establece la gratuidad universitaria y en 1953 que 
se eliminan los exámenes de ingreso. En términos numéricos el resultado es extraordinario: el sistema universitario argentino que tenía 47.000 estudiantes en 1945, pasa a tener 138.000 en 1955 (Cano, 1985).

El debate sobre quiénes pueden acceder a la educación superior, de todas maneras, no quedó saldado. Luego del Golpe de Estado de 1955 se derogaron las normativas que había elaborado el peronismo respecto a la educación superior y la forma de ingreso comenzó a depender de cada institución. Esto volvió a modificarse por un período breve de tiempo en 1973, con las importantes reformas llevadas adelante durante el gobierno de Héctor Cámpora, que configuraron una importante explosión en la matrícula universitaria. En este momento encontramos la primera experiencia de ingreso para mayores de 25 años en la Universidad Nacional de Luján. Esta experiencia fue breve, ya que con el Golpe de Estado de 1976 se dejó de inscribir estudiantes bajo esta modalidad.

Con la dictadura, se impusieron exámenes de ingreso en todas las Universidades y se aplicaron aranceles. Se trató del período de mayor reducción en la matrícula universitaria en los últimos 70 años: en 1975, el sistema universitario contaba con 487.389 estudiantes, y en 1980 con 386.7431.

La vuelta de la democracia en 1983 estuvo acompañada nuevamente por el ingreso irrestricto y la eliminación de los aranceles, lo que llevó a una nueva explosión de la matrícula universitaria, que llegó a contar con 700.00 estudiantes en 1986 (Buchbinder, 2005).

Se observa a partir de este repaso histórico como las políticas de acceso y admisión a la educación superior fueron cambiando a lo largo de todo el siglo $\mathrm{XX}$ en Argentina. Las políticas más restrictivas se llevaron adelante durante los 
gobiernos de facto, cuando se detienen los avances de la matrícula, aunque sólo la última dictadura logró que ésta descendiera. El ingreso irrestricto y la búsqueda del fortalecimiento de las Universidades como instituciones masivas marcó a todos los gobiernos democráticos desde 1949. No obstante, en la década de 1990 esta legitimidad democrática del acceso irrestricto estuvo en discusión: la ley universitaria sancionada en 1995 no planteaba este tema, dejando abierta la posibilidad tanto de exámenes eliminatorios, como de arancelamientos. Sin embargo, habilitó una nueva modalidad de ingreso, ajena hasta ese momento al marco normativo argentino: el ingreso para mayores de 25 años que no acrediten título secundario, pero que demuestren poseer experiencia laboral y/o preparación acorde a los estudios que deseen cursar. Esta herramienta, pensada como excepcional, tardó en ser implementada por las principales universidades del país, que presentaron resistencias.

Entre 2003 y 2015 hubo una serie de políticas que permitieron el crecimiento constante de la matrícula universitaria: fortalecimiento del presupuesto universitario que redundó en un crecimiento del sistema de becas y de las políticas de bienestar universitario, en el marco de un crecimiento general del sistema científico; la creación de nuevas universidades; la implementación de algunas medidas nacionales destinadas a promover estudios, como el caso de la beca PROGRESAR. Pero en lo que respecta a políticas de acceso, no hubo ninguna novedad hasta la reforma parcial de la Ley de Educación Superior en 2015. Por primera vez, la gratuidad y el ingreso irrestricto aparecían con fuerza de ley. La modalidad de ingreso para mayores de 25 no fue modificada en ningún aspecto.

Reseñamos rápidamente este proceso porque, aunque no podemos detenernos en detalle, nos parece importante para entender que el acceso a la 
educación superior en nuestro país estuvo en debate durante todo el siglo XX, y que diferentes concepciones acerca del mismo impactaron en la composición de las Universidades Nacionales.

\section{Saberes del trabajo e inclusión educativa}

El artículo de la Ley de Educación Superior que estamos analizando no estipulaba el ingreso irrestricto en su formulación original, el que, como vimos, se había constituido en la historia de nuestro país como una política de acceso central para favorecer la masividad de la educación superior. Pero sí establece la posibilidad de una forma de ingreso excepcional para quienes no acrediten el nivel de estudio correspondiente. Es decir: en el marco de un artículo que no garantizaba la herramienta histórica de democratización de la Universidad aparece la posibilidad de pensar un mecanismo novedoso de inclusión educativa.

En primer lugar, analizaremos qué tipo de saber se considera aceptable para cursar estudios superiores, y luego intentaremos indagar qué condiciones se deberían cumplir para que efectivamente se trate de una política de inclusión educativa.

La LES formula dos condiciones para poder ingresar a la Universidad sin acreditar estudios secundarios: aptitudes y conocimientos, por un lado; y preparación o experiencia laboral acorde a los estudios que se pretenden iniciar, por el otro.

Esta noción de preparación y/o experiencia laboral introduce un elemento novedoso. Contar con estudios secundarios es una forma de acreditar aptitudes y conocimientos, con lo cual el primero de los requisitos para permitir el ingreso de personas sin estudios secundarios está fuertemente asociado al 
tipo de conocimiento que se exige para ingresar en la Universidad en general. Pero el segundo requisito introduce la idea de que existe un tipo de saber, asociado a la experiencia laboral, que puede ser reconocido y valorado para comenzar los estudios universitarios.

Tomamos el concepto de saberes del trabajo de Ramón Roqueiro, quien lo define como:

Entendemos como saberes del trabajo a aquellos conocimientos complejos que las personas utilizan para la producción de bienes (tangibles e intangibles) y servicios a cambio de una remuneración (en dinero o en otros bienes) (Roqueiro, 2007, p. 60).

Se trata no solamente de una serie de conocimientos en sentido estricto, sino también de la capacidad de resolución de problemas, de aplicación de esos conocimientos en la práctica y finalmente de transmisión y producción de nuevos conocimientos a partir de la práctica. Podemos vincular el concepto de saberes del trabajo a la posibilidad de pensar en los sujetos que van a ingresar en la universidad no solamente desde una perspectiva individual, sino también social. Es en este sentido que Castronovo sostiene que "los estudiantes que ingresan por el Artículo 7 han realizado trayectorias de construcción social del conocimiento que le permitirían tener una visión menos individual y más social de sus talentos" (2009, p. 63).

Resulta interesante, que una ley sancionada en un contexto neoliberal, en el que los sujetos de la educación son centralmente entendidos de forma individual y deshistorizada, se incluya, aunque sea de forma excepcional, la posibilidad de reconocimiento de trayectorias variadas, donde no sean los logros escolares la única variable a tener en cuenta. En general, la relación entre universidad y trabajo fue pensada en clave unidireccional. Los estudios 
superiores forman graduados para el mundo del trabajo. Muy pocas veces aparece la posibilidad de pensar la relación inversa: en qué medida el trabajo forma para la Universidad. El artículo 7 de la LES abre la posibilidad de pensar entonces en una articulación más compleja entre trabajo y Universidad, "supone encontrar los modos de articulación entre dos tipos de saberes: los saberes de la experiencia y los saberes académicos" (Castronovo, 2009, p. 70). Este elemento interesante, puede resultar también problemático. En primer lugar, ¿de qué forma puede la Universidad acreditar esos saberes del trabajo como suficientes? Dicho de otra manera: cuáles son los mecanismos de conversión de saberes aprendidos en ámbitos distintos a la educación formal, a los criterios que establece la educación formal. Este problema tiene una enorme complejidad, pero en lo que respecta al tema de nuestro trabajo, podemos decir que cada reglamentación de los mecanismos de ingreso para mayores de 25 años sin título secundario, como es el caso de la Ordenanza n²55/02, constituye un intento de responder a esta cuestión.

Pero en segundo lugar aparece otro problema, y es que este reconocimiento de los saberes del trabajo puede implicar un requisito que termine afectando el carácter democratizador de esta iniciativa. Nos referimos a que esta modalidad de ingreso exige tener conocimientos y aptitudes suficientes, que es lo que se acredita también mediante estudios secundarios. Los saberes del trabajo pueden aparecer así como un requisito extra.

Creemos al respecto que es posible pensar este problema de otra manera. La Universidad puede incorporar estudiantes que no tengan una trayectoria formal completa, reconociendo para esto otro conjunto de saberes, vinculados a formas de educación no formal, educación informal, aprendizajes de la experiencia y saberes del trabajo. Es decir, es necesario contemplar, en los 
casos en que les aspirantes a ingresar a la Universidad no acrediten estudios secundarios, sus conocimientos y su experiencia. Pero estos elementos conocimientos y experiencia- no tienen que ser entendidos como requisitos separados a ser evaluados independientemente; sino como aspectos complementarios a la hora de analizar y evaluar sus capacidades con un criterio de inclusión y democratización educativa.

Entonces, que este artículo se convierta efectivamente en una herramienta de inclusión, depende de un trabajo de interpretación y reglamentación. Como veremos más adelante, en la mayoría de los casos, el carácter excepcional tuvo más peso en las reglamentaciones realizadas por las universidades que la búsqueda de reconocer otro tipo de trayectorias y de adquisición de saberes.

Cuando hablamos de inclusión, no nos referimos solamente a la masividad, que "se reduce a la ampliación del acceso" (Chiroleu, 2009, p.100) sino que partimos "del reconocimiento de que la sociedad no es homogénea y la diversidad constituye un componente fundamental que merece ser revalorizado." (Chiroleu, 2009, p.100).

La inclusión supone recuperar y revalorizar la diversidad; pero además tiene que dar cuenta de la desigualdad, por lo que su preocupación fundamental es la equidad en el acceso educativo: la búsqueda de que la Universidad sea accesible a todes, y en particular a quienes están en peores condiciones. En un país desigual como Argentina, la inclusión tiene que ver con "aplicar el principio de equidad vertical diseñando políticas públicas e institucionales que brinden un tratamiento diferencial a los que se encuentran en una situación inicial desigual" (Garcia de Fanelli, 2014, p. 4).

La importancia de la noción de inclusión se encuentra vinculada a otra que está consagrada en la modificación parcial que se produjo en 2015 de la Ley que 
estamos analizando: la educación superior es un derecho. Como tal, la obligación del Estado no es solamente tener una política de puertas abiertas para quienes cumplan los requisitos y deseen ingresar, sino trabajar activamente para garantizar la posibilidad del estudio a todas aquellas personas que lo deseen.

El artículo 7 de la LES, permite incorporar una herramienta de inclusión tal como lo hemos presentado en este apartado: una forma de incorporar sujetos que no pudieron completar el proceso de estudios formales obligatorios, reconociendo otras trayectorias y experiencias. Abre la posibilidad de realizar un pequeño aporte en el sentido de la construcción de una Universidad no sólo masiva, sino popular en su conformación.

No podemos desconocer, de todas maneras, que se trata de una posibilidad que la mayoría de las veces no se materializó. Las Universidades reglamentaron tarde esta forma de ingreso, y prácticamente no realizaron seguimientos de las trayectorias educativas de estos estudiantes ni una sistematización de estas experiencias. Es por eso que resulta fundamental analizar las formas que de hecho han tenido los intentos de implementar el ingreso para personas que no tienen estudios formales.

\section{La Ordenanza 255/01}

El Artículo 7 de la Ley de Educación Superior se encuentra reglamentado en nuestra Universidad por la Ordenanza 255, aprobada en 2001. Dicha ordenanza es la primera y única regulación al respecto que tiene esta casa de altos estudios.

En ella se establecen dos instancias que deberán cumplir quienes aspiran a ingresar bajo esta modalidad, llamadas respectivamente "Evaluación de 
antecedentes" y "Evaluación de aptitudes y conocimientos". En primer lugar cada aspirante deberá realizar una Solicitud de Evaluación en la Unidad Académica donde se dicte la carrera de su interés. Será la Facultad, mediante su Secretaría Académica, la encargada de determinar si posee preparación o experiencia laboral acorde. Luego, la Secretaría de Asuntos Académicos será la encargada de evaluar a aspirantes que envían las Unidades Académicas, para determinar si poseen las aptitudes y conocimientos necesarios, mediante exámenes individuales y escritos de Matemática, Lengua y Literatura, Ciencias Sociales (Historia y Geografía) y Ciencias Naturales (Biología, Física y Química). Cada uno de estos exámenes deberá aprobarse con una nota igual o superior a seis (6), teniendo derecho a recuperatorio de una evaluación quienes obtengan más de cuatro (4) en ella o una nota promedio igual o superior a seis (6), esto es, que la suma de las notas de las cuatro evaluaciones sea igual o superior a veinticuatro (24). De esta manera la Ordenanza busca cumplir los dos elementos presentes en la Ley de Educación Superior, interpretándolos explícitamente como elementos separados. Las facultades determinan la preparación o experiencia laboral, y la Secretaría de Asuntos Académicos de la UNLP las aptitudes y conocimientos.

La ordenanza determina que a quienes aprueben los cuatro exámenes se les extenderá un "Certificado de Aspirante Autorizado", con el que podrán inscribirse en la fecha regular de ingreso a las carreras de la UNLP. Este certificado servirá en reemplazo del título o analítico secundario, siendo necesario que les aspirantes autorizados presenten el resto de la documentación necesaria.

Establece además que la recepción de solicitudes de evaluación deberá realizarse durante la segunda quincena de agosto, habiendo tiempo hasta el 15 
de septiembre para elevar los resultados de la Evaluación de Antecedentes a la Secretaría de Asuntos Académicos. Entre el 20 de septiembre y el 10 de octubre deberán tomarse los cuatro exámenes, y entre el 20 de octubre y el 10 de noviembre los correspondientes recuperatorios. Además, se determina que los programas serán elaborados por la Secretaría de Asuntos Académicos y deberán ser aprobados por el Presidente de la UNLP, con una antelación no menor a seis meses respecto al inicio del período de presentación de las solicitudes de evaluación.

Como podemos observar, las dos instancias de evaluación que prevé la Ordenanza 255/01 están desarrolladas de forma muy desigual. Lo que corresponde a la Evaluación de Antecedentes presenta un alto grado de flexibilidad, permitiendo a cada Unidad Académica elaborar los mecanismos que considere pertinentes. En cambio, lo que respecta a la segunda parte se encuentra fuertemente reglamentado, con un marcado énfasis en la adquisición de conocimientos por sobre la evaluación de aptitudes. Esto es, se busca que les aspirantes posean conocimientos similares a los que estipula el diseño curricular de la educación secundaria en la Provincia de Buenos Aires, más que el desarrollo de aptitudes de resolución de problemas o construcción de razonamientos.

Realizaremos un análisis de la forma en que se aplicó esta Ordenanza en nuestra Universidad, tomando centralmente los años 2018 y 2019 , ya que son los años con mayor masividad y sobre los que existen más registros. En todos los casos, trabajaremos a partir de información propia de la Secretaría de Asuntos Académicos. 


\section{Aplicación de la Ordenanza 255/01 en 2018 y 2019}

El primer año de vigencia y aplicación de esta Ordenanza fue el 2002, cuando se elaboraron los programas que aún hoy se encuentran vigentes. En ese momento, cada Facultad tenía autonomía para establecer sus mecanismos de admisión, por lo que en algunos casos era necesario rendir un examen de ingreso luego de obtener el Certificado de Aspirante Autorizado.

La mayor parte de la legislación en la que se sostuvo esta Ordenanza cambió: se sancionó la Ley de Educación Nacional en 2006, que reformó la enseñanza media en nuestro país, y con ella también los diseños curriculares que servían de fundamento a los programas de cada uno de los exámenes. En 2008 se aprobó un nuevo Estatuto de la UNLP, donde se establece el carácter irrestricto del acceso a la Universidad y se fortalece la noción de educación como derecho. En el mismo sentido se produce una modificación parcial de la Ley de Educación Superior en 2015. Pese a todos estos cambios no sólo nunca fue modificada la Ordenanza, sino que tampoco lo fueron los programas de cada una de las materias, que son prorrogados año a año.

En 2018 se organizó la convocatoria para el ingreso de mayores de 25 que no presentan título secundario de la misma forma que se había realizado en años anteriores, difundiendo esta modalidad en la página de la UNLP y en algunas radios y portales informativos de la ciudad de La Plata. El resultado fue que 65 personas completaron la Solicitud de Evaluación. La Facultad más elegida fue Ciencias Jurídicas y Sociales, que tuvo 21 solicitudes de evaluación, el doble de la Facultad de Ciencias Médicas, elegida por 10 aspirantes. Le siguieron, en orden, la Facultad de Humanidades y Ciencias de la Educación, con 9; Ingeniería y Ciencias Económicas, con 5; y Ciencias Veterinarias, con 3. Hubo 
2 aspirantes en Informática, Ciencias Exactas, Arquitectura y Urbanismo, Psicología y Periodismo y Comunicación Social; y un solo aspirante en Trabajo Social y en Ciencias Naturales y Museo. No tuvieron inscriptos Ciencias Agrarias y Forestales, Ciencias Astronómicas y Geofísicas, Odontología ni Artes.

Si ordenamos a les aspirantes por carrera la diferencia resulta aún más profunda. Abogacía es la carrera que eligieron 21 aspirantes, seguida de lejos por Enfermería con 7 y por Contador Público por 4. Es decir, entre las 120 carreras que ofrece nuestra Casa de Altos Estudios, casi un tercio de las personas interesadas optó por la misma: Abogacía, una carrera que podríamos considerar larga (tiene una duración de 5 años) y tradicional.

Las Facultades utilizaron criterios flexibles para la evaluación de antecedentes:en general, se trató de entrevistas realizadas por la Secretaría Académica de cada Unidad Académica. Se privilegó de hecho la instancia de evaluación de aptitudes, y en este segundo momento la situación fue muy distinta: sólo 28 personas se presentaron a rendir al menos un examen, 4 aprobaron al menos un examen y dos personas aprobaron los cuatro, pudiendo ingresar bajo esta modalidad. Podemos ver en este gráfico el porcentaje de personas que rindieron, que aprobaron al menos un examen y quienes efectivamente lograron su certificado de aspirante autorizado, considerando como $100 \%$ a las 65 personas que pasaron la evaluación de antecedentes.

En el segundo año se buscó sostener el nivel de difusión alcanzado el primer año, garantizado a su vez un mayor acompañamiento que intentara mejorar la tasa de ingreso. Esto, considerando un primer balance provisorio: existe un universo de personas interesadas en acceder a la UNLP que no cuentan con 
título secundario, pero la forma de ingreso tal como ha estado planteada lo habilita de forma muy excepcional.

Para esto, se elaboraron pautas para rendir los exámenes con menor carga teórica y densidad bibliográfica, intentando ponderar aptitudes más que conocimientos. Las pautas enfatizaban en contenidos procedimentales por sobre los contenidos conceptuales, intentando evaluar la capacidad de elaborar razonamientos específicos de cada una de las disciplinas evaluadas.

A la vez, se trabajó con un cuerpo de docentes contratados específicamente para el programa, encargados de elaborar las pautas, seleccionar la bibliografía y evaluar, pero también de brindar clases de consulta semanales durante cuatro semanas previas al examen. Además, se puso al alcance de les aspirantes toda la bibliografía necesaria.

Estas modificaciones produjeron algunos cambios. En primer lugar, la cantidad de solicitudes de evaluación se elevó un 45\% pasando de 65 a 94. La cantidad de personas que rindieron al menos un examen pasó de 28 a 46, lo que significa un crecimiento de $64 \%$. Y la cantidad de personas que aprobaron y quedaron en condiciones de ingresar se multiplicó dieciocho veces, pasando de 2 a 36. Más aún, las 45 personas que rindieron aprobaron al menos un examen. Seguimos viendo un descenso pronunciado entre las personas que se anotan y las que rinden, y entre las que rinden y las que aprueban, pero la diferencia con 2018 es notable. Veamos el mismo gráfico que presentamos para el 2018, pero en el caso del 2019:

Si partimos de la cantidad de estudiantes que rindieron, aunque sea un examen y no de todas las personas que se inscribieron, la diferencia entre 2018 y 2019 es aún más clara. Se pasó de una tasa de un 7,1\% de aspirantes aprobados a un $80 \%$. 
Como ya mencionamos, estos importantes cambios se lograron clarificando las pautas, orientando la evaluación más hacia aptitudes y capacidad de resolución de problemas que a conocimientos y mejorando el acompañamiento y el apoyo a les aspirantes.

Respecto a las Unidades Académicas y carreras más elegidas en 2019, el esquema replica con pequeños cambios lo sucedido en 2018.

Como podemos ver, Ciencias Jurídicas y Sociales siguió siendo la Facultad más elegida, seguida por Ciencias Médicas y Humanidades y Ciencias de la Educación. Podemos observar un crecimiento de Psicología, que pasó de 2 a 11 aspirantes; y de Trabajo Social, que pasó de 1 a 7.

Respecto al género, en 2018 se anotaron 36 mujeres y en 2019 50, frente a 30 y 44 varones respectivamente, lo que nos habla de números parejos. Tampoco encontramos un fuerte impacto de este indicador en estudiantes que aprobaron los cuatro exámenes, siendo 19 mujeres frente a 17 varones en el 2019. En el 2018 sólo ingresaron 2 varones.

El último dato importante a tener en cuenta es el que respecta a las edades: el promedio de edad en 2018 era aproximadamente de 35 años y la mediana de 33 años, con una amplia variedad de edades que iban desde los 24 años (personas que iban a cumplir 25 antes del ciclo lectivo siguiente) y los 56 años. En 2019 la edad promedio sube apenas, con un promedio de 37 años y una mediana de 35. Por lo que podemos decir que la mayoría de las personas que se inscribieron tuvieron su edad escolar durante los años de aplicación y funcionamiento de la Ley Federal de Educación, sancionada en 1993. Sin embargo, aún es pronto para saber si la obligatoriedad de la educación secundaria, sancionada con la Ley de Educación Nacional en 2006 va a tener 
repercusiones en la cantidad de aspirantes a ingresar a la Universidad sin título secundario.

No existe información sistematizada que permita conocer la cantidad de estudiantes que trabajaban en áreas relacionadas, ni cuáles eran los móviles que les propies estudiantes explicitaban al momento de inscribirse.

\section{Reflexiones finales}

La Universidad Nacional de La Plata tiene una concepción de la educación superior como derecho sostenida en su Estatuto y en cada uno de sus planes estratégicos desde 2004. En este sentido ha desarrollado múltiples programas que apuestan a fomentar el ingreso, la permanencia y el egreso, con diferentes políticas de inclusión educativa. Esto se profundizó a partir del Plan Estratégico 2018-2022. Sin embargo, la dimensión específica del ingreso de mayores de 25 años sin título secundario recién comienza a ser abordada en su integralidad. No existen registros históricos de quienes ingresaron bajo esta modalidad que permitan realizar un seguimiento de sus recorridos educativos, analizar la tasa de deserción y los motivos de la misma. Tampoco existen registros que nos permitan conocer los recorridos previos de quienes deciden iniciar la carrera bajo esta modalidad, así como sus intereses y motivaciones.

Esta falta de registros es común a casi todas las Universidades Nacionales de nuestro país, debido a que el tema que nos ocupa se entendió siempre como una forma de ingreso excepcional. Sin embargo, los pocos registros que existen nos permiten pensar que en todas las Universidades Nacionales donde esta posibilidad se difundió, tuvo aspirantes.

En los últimos dos años en nuestra Casa de Altos Estudios se observa algo que también es visible en todas las otras experiencias reseñadas, tanto previas 
a la Ley de Educación Superior como referidas a la aplicación de su artículo 7 en diferentes Universidades Nacionales: cuando existe una política de acompañamiento a los procesos de estudios, espacios de consulta y taller, la diferencia de resultados en los exámenes es notable. En muchos casos se trata de personas que no tienen dinámica de lectura y escritura académica hace muchos años, con lo que este apoyo resulta fundamental para poder recuperar formas de razonamiento propias de la educación formal de nivel universitario.

Además, las evaluaciones centradas en contenidos, buscando reproducir los contenidos mínimos de toda la educación secundaria en una instancia de examen final, resultan en bibliografías extensas, difíciles de abarcar en pocos meses. En ese sentido, son interesantes las experiencias que entienden que de lo que se trata es de comprender textos universitarios, poder producirlos, así como interpretar datos y desarrollar razonamientos acordes a la educación superior.

Un elemento mencionado durante este trabajo, que resulta en una dificultad específica para pensar en reformas de esta Ordenanza está vinculado a que hoy en nuestro país la finalización del nivel medio es obligatoria por ley, por lo que los esfuerzos del Estado Nacional y Provincial están puestos en generar mayores niveles de graduación, para lo que se realizaron importantes programas, como el caso del FINes. Resultaría problemático que esta modalidad de ingreso a la Universidad atente contra esta posibilidad, ya que su aprobación no significa la finalización de los estudios secundarios. Es por eso que creemos que cualquier proyecto que se plantee debe dejar explícito que se seguirá trabajando para buscar que aumente la cantidad de egresados del nivel secundario y quienes se encarguen de la orientación y apoyo de les mayores 
de 25 años deben conocer los programas que existen y poder orientarles al respecto.

Considerando todo lo planteado, creemos que hay condiciones suficientes para fortalecer en lo inmediato los procesos de acompañamiento del ingreso de personas mayores de 25 años, tanto en el momento previo al ingreso como durante los primeros momentos de la carrera universitaria. A su vez, que resulta necesario el registro y sistematización de las expectativas y experiencias de quienes ingresan bajo esta modalidad.

A través de este trabajo hemos dejado planteadas las principales características que creemos que debe asumir una propuesta de nueva Ordenanza para reglamentar el artículo 7 de la Ley de Educación Superior de modo que ella quede articulada con el proyecto político-pedagógico que defiende la Universidad Nacional de La Plata: Universidad masiva, inclusiva y popular.

\section{Bibliografía}

- Belnicoff, María Antonia. (2008). En torno a la propuesta de Reglamentación del Artículo No7 de la Ley Nacional de Educación Superior [en Línea]. GOBIERNO DE LA CIUDAD DE BUENOS AIRES MINISTERIO DE EDUCACION - DIRECCIÓN GENERAL DE PLANEAMIENTO. Disponible en: https://www.buenosaires.gob.ar/sites/gcaba/files/aplicacion articulo 7.p $\underline{\mathrm{df}}$

- Buchbinder, P. (2005). Historia de las Universidades Argentinas. Buenos Aires: Sudamericana. 
- Cano, D. (1985). La educación superior en la Argentina. Buenos Aires: FLACSO.

- Castronovo, Adela. (2009). El ingreso a la Universidad de los mayores de 25 años sin título secundario contemplado en el artículo 7 de la Ley de Educación Superior [en Línea]. Universidad Nacional de Lanús. Departamento de Humanidades y Artes. Disponible en: http://www.repositoriojmr.unla.edu.ar/descarga/Tesis/MAMIC/031716 C astronovo.pdf

- Chiroleu, A. (2009). La democratización del acceso a la universidad: de la ampliación de oportunidad a la inclusión. A 90 años de la Reforma Universitaria: memorias del pasado y sentidos del presente, 99-116.

- Chiroleu, Adriana: Políticas de Admisión a la Universidad. Una aproximación a las experiencias de Brasil y Argentina. Revista de Estudios Sociales Año 2 No 2. 10 semestre de 1992. 167-183.

- Estatuto de la Universidad Nacional de La Plata. (Asamblea Universitaria de la Universidad Nacional de La Plata). 11 de octubre de 2008.

- García de Fanelli, Ana (2014). Inclusión social en la Educación Superior argentina: Indicadores y políticas en torno al acceso y a la graduación, vol.7, n.2, pp.124-151. ISSN 1688-7468. Recuperado de: http://www.cedes.org/publicaciones/documentos/EdSup/2014/10669.pdf

- Ordenanza 3 de 2015 (Consejo Superior de la Universidad Nacional de Córdoba). Reemplaza a la Resolución 409 de 2000. 7 de julio de 2015.

- Ordenanza 255 de 2001 (Consejo Superior de la Universidad Nacional de La Plata). Reglamenta el proceso de admisión de los mayores de 25 años que no acrediten aprobación del nivel medio. 
- Oviedo de Ignazzi, R. y Salibián, A. (1978). Análisis de una experiencia novedosa en la enseñanza de la ecología, Revista Ecología N³: 9-17.

- Pain, A. (1992) Educación informal. El potencial educativo de las situaciones cotidianas. Buenos Aires, Nueva Visión.

- Plan estratégico de la Universidad de La Plata 2014-2018 1 era. Edición - Mayo de 2014.

- Plan estratégico de la Universidad de La Plata 2018-2022 1 era. Edición - Mayo de 2018.

- Rinesi, E; Soprano, G y Suasnabar, C (comp) (2005) Universidad: reformas y desafíos. Dilemas de la educación superior en la Argentina y el Brasil. Buenos Aires, UNGS y Prometeo.

- Roqueiro, R. (2007). "Los saberes del trabajo", en Garcés L. ¿De la escuela al trabajo? (2007). Buenos Aires, Ediciones del signo.

- Rovelli, L. (2009). Del plan a la política de creación de nuevas universidades nacionales en Argentina: la expansión institucional de los años 70 revisitada. Temas y Debates, 117-137.

- Sánchez, L. (1969) La universidad actual y la rebelión juvenil. Buenos Aires, Editorial Losada. 